\title{
An Explicit Functional Process Solution to a Stochastic Partial Differential Equation with Applications to Nonlinear Filtering.
}

\author{
Fred Espen Benth \\ Department of Mathematics \\ University of Oslo \\ Box 1053 Blindern, N-0316 Oslo \\ Norway
}

\begin{abstract}
We find an explicit functional process solution to a stochastic partial differential equation which arises in nonlinear filtering theory. The solution is constructed using the Hermite transform.
\end{abstract}

Key Words: Stochastic partial differential equations, Hermite transform, white noise, nonlinear filtering.

\section{Introduction.}

This paper considers the stochastic Cauchy problem given by

$$
\begin{gathered}
d \phi_{t}(x, \omega)=\left(L_{x} \phi_{t}(x, \omega)-c(t, x) \phi_{t}(x, \omega)\right) d t+h(t, x) \phi_{t}(x, \omega) d B_{t} \\
\phi_{0}(x, \omega)=p_{0}(x)
\end{gathered}
$$

where $L_{x}$ is an infinitesimal generator associated to an Ito diffusion. $B_{t}$ is a Brownian motion. We will have a look at a solution concept called a functional process solution, which is a weak solution concept to this stochastic partial differential equation. We substitute the Ito integral part of (1) with a Lebesgue integral involving the Wick product together with a white noise process, and find the solution to this problem instead.

The problem of solving (1) and (2) comes from nonlinear filtering theory. The linear filtering problem has the well-known Kalman-Bucy filter as its solution, which is an ordinary stochastic differential equation. The nonlinear case, however, leads to finding the unnormalised probability density, which satisfies a stochastic partial differential equation. This equation is in filtering theory known as the Wong-Zakai equation and equals (1) when $L_{x}-c=A^{*} . A^{*}$ is the adjoint to the infinitesimal generator associated to the system equation in the filtering problem. Pardoux [12] and Gyöngy \& Krylov [5] have worked out general existence and uniqueness results for (1) and (2). An explicit solution of the problem, however, has, as far as I know, never been found. In this article, we use the Hermite transform to "translate" our stochastic partial differential equation into an "ordinary" partial differential equation, which has a stochastic version of its solution. Invers Hermite transform then produces a solution candidate. In section 4 we demonstrate how this technique works. The technique has been used for several other problems, see for instance [7].

We start in section 2 with some mathematical preliminaries which we are going to use troughout this paper. We also define the functional process solution concept. Section 3 considers 
expectation in $(\mathcal{S})^{*}$ and some results for the Wick product which will be useful in section 5 . The last section contains the proof of the functional process solution of the stochastic Cauchy problem.

\section{Some Mathematical Preliminaries.}

This brief introduction of some aspects of the white noise theory is mostly taken from [4]. For a more complete account, see [6].

We start by recalling some of the basic definitions and features of the white noise probability space. As usual, let $\mathcal{S}^{\prime}=\mathcal{S}^{\prime}\left(\mathbb{R}^{d}\right)$ denote the space of tempered distributions on $\mathbb{R}^{d}$, which is the dual of the well-known Schwartz space $\mathcal{S}\left(\mathbb{R}^{d}\right)$. By the Bochner-Minlos theorem there exists a measure $\mu$ on $\mathcal{S}^{\prime}$ such that

$$
\int_{\mathcal{S}^{\prime}} e^{i\langle\omega, \phi\rangle} d \mu(\omega)=e^{-\frac{1}{2}\|\phi\|^{2}}, \phi \in \mathcal{S}\left(\mathbb{R}^{d}\right)
$$

where $\|\cdot\|$ is the norm in $L^{2}\left(\mathbb{R}^{d}\right)$. This measure corresponds to the bilinear form

$$
\mathcal{E}(\phi, \psi)=\int_{\mathbb{R}^{d}} \phi \psi d x ; \phi, \psi \in \mathcal{S}\left(\mathbb{R}^{d}\right)
$$

Let $\mathcal{B}$ denote the Borel sets on $\mathcal{S}^{\prime}$ (equipped with the weak star topology). Then the triple $\left(\mathcal{S}^{\prime}\left(\mathbb{R}^{d}\right), \mathcal{B}, \mu\right)$ is called the white noise probability space.

Definition $1 A$ white noise process is a map

$$
W: \mathcal{S} \times \mathcal{S}^{\prime} \rightarrow \mathbb{R}
$$

given by

$$
W(\phi, \omega)=W_{\phi}(\omega)=\langle\omega, \phi\rangle, \omega \in \mathcal{S}^{\prime}, \phi \in \mathcal{S}
$$

Since $\mathcal{S}$ is dense in $L^{2}$, we can define $\langle\omega, \phi\rangle$ for $\phi \in L^{2}$ by

$$
\langle\omega, \phi\rangle=\lim _{n \rightarrow \infty}\left\langle\omega, \phi_{n}\right\rangle
$$

where $\phi_{n} \in \mathcal{S}^{\prime}$ is a sequence converging to $\phi \in L^{2}$. In particular, if we define

$$
\tilde{B}_{x}(\omega):=\tilde{B}_{x_{1}, \ldots, x_{d}}(\omega):=\left\langle\omega, \mathcal{X}_{\left[0, x_{1}\right] \times \ldots \times\left[0, x_{d}\right]}(.)\right\rangle
$$

then $\tilde{B}_{x}$ has an x-continuous version $B_{x}$ which becomes a d-parameter Brownian motion.

The d-parameter Wiener-Ito integral of $\phi \in L^{2}$ is defined by

$$
\int_{\mathbb{R}^{d}} \phi(y) d B_{y}(\omega)=\langle\omega, \phi\rangle
$$

The left hand side coincides with the Ito integral if $\operatorname{supp}(\phi) \subset[0, \infty)$. (See [11], p.4). Of special interest now will be the space $L^{2}\left(\mathcal{S}^{\prime}\left(\mathbb{R}^{d}\right), \mu\right)$ or $L^{2}(\mu)$ for short. The Wiener-Ito chaos expansion theorem says that every $F \in L^{2}(\mu)$ has the form

$$
F(\omega)=\sum_{n=0}^{\infty} \int_{\left(\mathbb{R}^{d}\right)^{n}} f_{n}(u) d B_{u}^{\otimes n}(\omega)
$$

where $f_{n} \in L^{2}\left(\mathbb{R}^{n d}\right)$ and $f_{n}$ is symmetric in all its $n d$ variables (in the sense that $f_{n}\left(u_{\sigma_{1}}, \ldots, u_{\sigma_{n d}}\right)=$ $f_{n}\left(u_{1}, \ldots, u_{n d}\right)$ for all permutations $\left.\sigma.\right)$. The right hand side of $(7)$ are the multiple Ito integrals. With $F, f_{n}$ as in (7) we have

$$
\|F\|_{L^{2}(\mu)}^{2}=\sum_{n=0}^{\infty} n !\left\|f_{n}\right\|_{L^{2}\left(\mathbb{R}^{n d}\right)}^{2}
$$


There is an equivalent expansion of $F \in L^{2}(\mu)$ in terms of the Hermite polynomials

$$
h_{n}(x)=(-1)^{n} e^{\frac{x^{2}}{2}} \frac{d^{n}}{d x^{n}}\left(e^{-\frac{x^{2}}{2}}\right) ; n=0,1,2, \ldots
$$

We now explain this more closely. Define the Hermite function of order $n$ as $\xi_{n}(x)$

$$
\xi_{n}(x)=\pi^{-1 / 4}((n-1) !)^{-1 / 2} e^{-\frac{x^{2}}{2}} h_{n-1}(\sqrt{2} x)
$$

where $x \in \mathbb{R}, n=0,1,2, \ldots\left\{\left\{\xi_{n}\right\}_{n=1}^{\infty}\right.$ forms an orthonormal basis for $L^{2}(\mathbb{R})$. Therefore the family $\left\{e_{\alpha}\right\}$ of tensor products

$$
e_{\alpha}:=e_{\alpha_{1}, \ldots, \alpha_{d}}:=\xi_{\alpha_{1}} \otimes \ldots \otimes \xi_{\alpha_{d}}
$$

(where $\alpha$ denotes the multi-index $\left(\alpha_{1}, \ldots, \alpha_{d}\right)$ ) forms an orthonormal basis for $L^{2}\left(\mathbb{R}^{d}\right)$. Assume that the family of all multi-indices $\beta=\left(\beta_{1}, \ldots, \beta_{d}\right)$ is given a fixed ordering

$$
\left(\beta^{(1)}, \beta^{(2)}, \ldots, \beta^{(n)}, \ldots\right)
$$

where $\beta^{(k)}=\left(\beta_{1}^{(k)}, \ldots, \beta_{d}^{(k)}\right)$. Put

$$
e_{n}=e_{\beta(n)} ; n=1,2, \ldots
$$

Let $\alpha=\left(\alpha_{1}, \ldots, \alpha_{m}\right)$ be a multi-index. It was shown by Ito that

$$
\int_{\left(\mathbb{B}^{d}\right)^{n}} e_{1}^{\hat{\otimes} \alpha_{1}} \hat{\otimes} \ldots \hat{\otimes} e_{m}^{\hat{\otimes} \alpha_{m}} d B^{\hat{\otimes} n}=\prod_{j=1}^{m} h_{\alpha_{j}}\left(\theta_{j}\right)
$$

where $\theta_{j}(\omega)=\int_{\mathbb{R}^{d}} e_{j}(x) d B_{x}(\omega), n=|\alpha|$ and $\hat{\otimes}$ denotes the symmetrized tensor product, so that, e.g., $f \hat{\otimes} g(x, y)=\frac{1}{2}[f(x) g(y)+f(y) g(x)]$ if $x, y \in \mathbb{R}$ and similarly for more than two variables. If we define, for each multi-index $\alpha=\left(\alpha_{1}, \ldots, \alpha_{m}\right)$,

$$
H_{\alpha}(\omega)=\prod_{j=1}^{m} h_{\alpha_{j}}\left(\theta_{j}\right)
$$

then we see that (11) can be written

$$
\int_{\left(\mathbb{R}^{d}\right)^{n}} e^{\hat{\otimes} \alpha} d B^{\otimes|\alpha|}=H_{\alpha}(\omega)
$$

using multi-index notation: $e^{\hat{\otimes} \alpha}=e_{1}^{\hat{\otimes} \alpha_{1}} \hat{\otimes} \ldots \hat{\otimes} e_{m}^{\hat{\otimes} \alpha_{m}}$ if $e=\left(e_{1}, e_{2}, \ldots\right)$. Since the family $\left\{e^{\hat{\otimes} \alpha} ;|\alpha|=n\right\}$ forms an orthonormal basis for the symmetric functions in $L^{2}\left(\left(\mathbb{R}^{d}\right)^{n}\right)$, we see by combining (7) and (13) that we have the representation

$$
F(\omega)=\sum_{\alpha} c_{\alpha} H_{\alpha}(\omega)
$$

(the sum being taken over all multi-indeces $\alpha$ of nonnegative integers). Morover, it can be proved that

$$
\|F\|_{L^{2}(\mu)}^{2}=\sum_{\alpha} \alpha ! c_{\alpha}^{2}
$$

where $\alpha !=\alpha_{1} ! \ldots \alpha_{m} !$. Using the expansion we have developed, the white noise process becomes

$$
W_{\psi}(\omega)=\int_{\mathbb{I}} \psi(x) d B_{x}(\omega)=\sum_{k=1}^{\infty}\left(\psi, e_{k}\right) H_{\epsilon_{k}}(\omega)
$$


where $\epsilon_{k}$ is the multi-index with 1 on the k'th coordinate, and zeros everywhere else. $(\cdot, \cdot)$ is the innerproduct in $L^{2}$.

There is a subspace of $L^{2}(\mu)$ which in some sense corresponds to the Schwartz subspace $\mathcal{S}\left(\mathbb{R}^{d}\right)$ of $L^{2}\left(\mathbb{R}^{d}\right)$. This space is called the Hida test function space and is denoted $(\mathcal{S})$ (see [6] for the construction of this space). Using a characterization due to Zhang in [13], a simple description of $(\mathcal{S})$ can be given as follows:

Definition 2 Let $F \in L^{2}(\mu)$ have the chaos expansion

$$
F(\omega)=\sum_{\alpha} c_{\alpha} H_{\alpha}(\omega)
$$

Then $F$ is a Hida test function, i.e. $F \in(\mathcal{S})$, if

$$
\sup _{\alpha} c_{\alpha}^{2} \alpha !(2 \mathbb{N})^{\alpha k}<\infty \forall \text { natural numbers } k<\infty
$$

where

$$
(2 \mathbb{N})^{\alpha}:=\prod_{j=1}^{m}\left(2^{d} \beta_{1}^{(j)} \ldots \beta_{d}^{(j)}\right)^{\alpha_{j}} \text { if } \alpha=\left(\alpha_{1}, \ldots, \alpha_{m}\right)
$$

The topological dual of $(\mathcal{S})$, denoted $(\mathcal{S})^{*}$, is called the Hida Distribution space. Zhang has in [13] a nice characterization of this space, too.

Theorem $3 A$ Hida distibution $G$ is a formal series

$$
G=\sum_{\alpha} b_{\alpha} H_{\alpha}
$$

where

$$
\sup _{\alpha} b_{\alpha}^{2} \alpha !\left((2 \mathbb{N})^{-\alpha}\right)^{q}<\infty \text { for some } q>0
$$

If $G \in(\mathcal{S})^{*}$ is given by (19) and $F \in(\mathcal{S})$ is given by (14), the action of $G$ on $F$ is given by

$$
\langle G, F\rangle=\sum_{\alpha} \alpha ! b_{\alpha} c_{\alpha}
$$

Note that no assumptions are made regarding the convergence of the formal series in (19).

We can in a natural way regard $L^{2}(\mu)$ as a subspace of $(\mathcal{S})^{*}$. In particular, if $X \in L^{2}(\mu)$ then by (21) the action of $X$ on $F \in(\mathcal{S})$ is given by

$$
\langle X, F\rangle=E[X \cdot F]
$$

Hence, the triple

$$
(\mathcal{S}) \subset L^{2}(\mu) \subset(\mathcal{S})^{*}
$$

An important example of an element in $(\mathcal{S})^{*}$ is the singular white noise, defined as

$$
W_{t}(\omega)=\sum_{k=1}^{\infty} e_{k}(t) H_{\epsilon_{k}}(\omega)=\sum_{k=1}^{\infty} e_{k}(t) h_{1}\left(\theta_{k}\right)
$$

Using estimates on $\xi_{k}$ from $\left[8\right.$, p.571] we can show that $W_{t}$ belongs to $(\mathcal{S})^{*}$. The important Wick product of two Hida distributions $F, G$ is defined as 
Definition 4 Let $F=\sum_{\alpha} a_{\alpha} H_{\alpha}$ and $G=\sum_{\beta} b_{\beta} H_{\beta}$ be two elements of $(\mathcal{S})^{*}$. Then the Wick product of $F$ and $G$ is the element $F \diamond G$ in $(\mathcal{S})^{*}$ given by

$$
F \diamond G=\sum_{\alpha, \beta} a_{\alpha} b_{\beta} H_{\alpha+\beta}
$$

It can be shown that the Wick product is closed in $(\mathcal{S})$, i.e.

$$
f, g \in(\mathcal{S}) \Rightarrow f \diamond g \in(\mathcal{S})
$$

This is not the case for two elements in $L^{2}(\mu)$. The Wick product of $F, G \in L^{2}(\mu)$ may fail to be in $L^{2}(\mu)$. Hence, the product must be handled with care when applied.

Using the Wick product, we can construct the Wick exponential. If $X \in L^{1}(\mu)$, define

$$
\operatorname{Exp} X:=\sum_{n=0}^{\infty} \frac{1}{n !} X^{\diamond n}
$$

when all the Wick powers of $X$ exist and the series converges in $L^{1}(\mu)$. (For the definition of the Wick product in $L^{1}(\mu)$, see [4]). In analogue with the classical exponential, we have the rule

$$
\operatorname{Exp}(X+Y)=\operatorname{Exp} X \diamond E x p Y
$$

when defined. The so-called positive noise can be constructed by the Wick exponential of a white noise process, $W_{\psi}$. For such processes there exist a connection between the Wick exponential and the classical exponential. The identity

$$
\sum_{n=0}^{\infty} h_{n}(x) \frac{t^{n}}{n !}=e^{t x-\frac{1}{2} t^{2}}
$$

for the Hermite polynomials, together with (11) give

$$
E x p W_{\psi}=\exp \left(W_{\psi}-\frac{1}{2}\|\psi\|^{2}\right)
$$

From Theorem 4.13 in [6], we have that the algebra generated by $\exp \left(\alpha W_{\psi}\right)$, where $\alpha \in \mathbb{C}$, is a subset of $(\mathcal{S})$. Hence it follows directly from the relation between the Wick exponential and the classical exponential that

$$
E x p W_{\psi} \in(\mathcal{S})
$$

Thus

$$
\operatorname{Exp} W_{\psi} \in L^{p}(\mu), \forall p \in<1, \infty>
$$

On the space $(\mathcal{S})^{*}$ there is a transform which enables us to translate stochastic equations into deterministic equations. This transform is called the Hermite transform, and is defined by:

Definition 5 Let $F \in(\mathcal{S})^{*}$ have the chaos expansion

$$
F=\sum_{\alpha} c_{\alpha} H_{\alpha}
$$

Then the Hermite transform of $F$ is

$$
\mathcal{H} F:=\tilde{F}:=\sum_{\alpha} c_{\alpha} z^{\alpha}
$$

where $z$ is a finite sequence in $\mathbb{C}$, i.e. $z \in \mathbb{C}_{0}^{\mathbb{N}}$, and $z^{\alpha}=z_{1}^{\alpha_{1}} \ldots z_{m}^{\alpha_{m}}$. 
One important property of the Hermite transform is that it changes Wick products into ordinary products:

Theorem 6 Let $F, G \in(\mathcal{S})^{*}$. Then

$$
\mathcal{H}(F \diamond G)=\mathcal{H} F \cdot \mathcal{H} G
$$

where the product on the right hand side is the usual complex product.

We will in the rest of the article only consider the case when $d=1$, i.e. $\mathcal{S}=\mathcal{S}(\mathbb{R})$ and $\mathcal{S}^{\prime}=\mathcal{S}^{\prime}(\mathbb{R})$. Hence, we get

$$
e_{k}(t)=\xi_{k}(t)
$$

We end this section by stating what we will mean by the functional process solution concept:

Definition 7 A map $\phi: C_{c}^{\infty} \times[0, T] \times \mathbb{R} \times \mathcal{S}^{\prime} \rightarrow \mathbb{R}$ is a functional process solution of (1) and (2) if $\phi_{t}^{\psi}(x, \cdot):=\phi(\psi, t, x, \cdot) \in L^{p}(\mu)$ for a $p \geq 1$ and

$$
\phi_{t}^{\psi}(x, \omega)=p_{0}(x)+\int_{0}^{t}\left(L_{x}-c(t, x)\right) \phi_{s}^{\psi}(x, \omega) d s+\int_{0}^{t} h(s, x) \phi_{s}^{\psi}(x, \omega) \diamond W_{\psi_{s}}(\omega) d s
$$

$\forall \psi \in C_{c}^{\infty}$, where $\psi_{s}(t)=\psi(t-s)$.

\section{$3 \quad$ Expectation In $(\mathcal{S})^{*}$.}

The proof of the functional process solution of (1) and (2), involves working with expectations of the Wick product of two processes. In this section, we will consider the problem of "moving a Wick product outside an expectation", i.e. investigate under what condodtions the Wick product commutes with the expectation. This will become easy in the context of expectation in $(\mathcal{S})^{*}$.

In section 5 we will apply these results on

$$
\operatorname{Exp} W_{\gamma(\eta)}(\omega) \diamond W_{\psi}(\omega) \in(\mathcal{S})
$$

Note here that $\gamma$ is assumed to be a stochastic variable too, dependent on $\eta \in \mathcal{S}^{\prime} . W_{\gamma(\eta)}(\omega)$ is then a map from $\mathcal{S}^{\prime} \times \mathcal{S}^{\prime}$ into $\mathbb{R}$, defined as

$$
(\eta, \omega) \rightarrow W_{\gamma(\eta)}(\omega)=\int_{\mathbb{R}} \gamma(\eta, s) d B_{s}(\omega)
$$

For each fixed $\eta,(29)$ is in $(\mathcal{S})$ as a function of $\omega$. At the end of this section we will show that $(29)$ is in $L^{2}(\mu)$ as a function of $\eta$.

Define the expectation of an $(\mathcal{S})^{*}$-element in the following way:

Definition 8 If $F: \mathcal{S}^{\prime} \rightarrow(\mathcal{S})^{*}$ is such that

$$
\langle F(\eta), \psi\rangle \in L^{1}(\mu), \forall \psi \in(\mathcal{S})
$$

then we define the unique $(\mathcal{S})^{*}$-element $\mathbb{E}[F(\eta)]$ to be

$$
\langle E[F(\eta)], \psi\rangle=E[\langle F(\eta), \psi\rangle]
$$

(The existence of $\mathbb{E}[F(\eta)]$ as a $(\mathcal{S})^{*}$-element can be proven by using the proof in Prop. 8.1, [6]). The reader should note that in the case of

$$
F: \mathcal{S}^{\prime} \rightarrow L^{2}(\mu)
$$

then $F$ is a stochastic variable on the product space $\mathcal{S}^{\prime} \times \mathcal{S}^{\prime}, F(\eta, \omega)$. We have the following situation:

$$
\left\langle\mathbf{E}_{\eta}[F(\eta, \cdot)], \psi\right\rangle=E_{\eta}[\langle F(\eta, \cdot), \psi\rangle]=E_{\eta}\left[E_{\omega}[F(\eta, \omega) \cdot \psi(\omega)]\right]
$$

Now we take a look at some important properties of this expectation: 
Proposition 9 Let $F: \mathcal{S}^{\prime} \times \mathcal{S}^{\prime} \rightarrow \mathbb{R}$. Assume $F(\cdot, \omega) \in L^{1}(\mu), F(\eta, \cdot) \in L^{2}(\mu)$ and $E_{\eta}\left[F^{2}(\eta, \cdot)\right] \in$ $L^{1}(\mu)$. Then

$$
\mathbb{E}_{\eta}[F(\eta, \omega)]=E_{\eta}[F(\eta, \omega)]
$$

Proof: We show that

$$
\left\langle E_{\eta}[F(\eta, \omega)], \psi\right\rangle=\left\langle\mathbf{E}_{\eta}[F(\eta, \omega)], \psi\right\rangle, \forall \psi \in(\mathcal{S})
$$

By the Hőlder inequality, we have

$$
E_{\omega}\left[\left(E_{\eta}[|F(\eta, \omega)|]\right)^{2}\right] \leq E_{\omega}\left[E_{\eta}\left[|F(\eta, \omega)|^{2}\right]\right]<\infty
$$

Hence

$$
E_{\eta}[F(\eta, .)] \in L^{2}(\mu)
$$

This implies

$$
\left\langle E_{\eta}[F(\eta, \cdot)], \psi\right\rangle=E_{\omega}\left[E_{\eta}[F(\eta, \omega)] \cdot \psi(\omega)\right]=E_{\omega}\left[E_{\eta}[F(\eta, \omega) \cdot \psi(\omega)]\right]
$$

We show that

$$
E_{\omega}\left[E_{\eta}[F \cdot \psi]\right]=E_{\eta}\left[E_{\omega}[F \cdot \psi]\right]
$$

The Tonelli theorem and Hőlder inequality give

$$
\begin{aligned}
& E_{\eta, \omega}\left[|F \cdot \psi|^{2}\right]=E_{\eta}\left[E_{\omega}\left[|F \cdot \psi|^{2}\right]\right] \leq E_{\eta}\left[E_{\omega}\left[F^{2}\right] \cdot E_{\omega}\left[\psi^{2}\right]\right] \\
= & \|\psi\|_{L^{2}(\mu)}^{2} E_{\eta}\left[E_{\omega}\left[F^{2}\right]\right]=\|\psi\|_{L^{2}(\mu)}^{2} \cdot E_{\omega}\left[E_{\eta}\left[F(\eta, \omega)^{2}\right]\right]<\infty
\end{aligned}
$$

which implies

$$
F(\eta, \omega) \cdot \psi(\omega) \in L^{2}(\mu \times \mu) \subset L^{1}(\mu \times \mu)
$$

Hence by the Fubini theorem, we can interchange $E_{\omega}$ and $E_{\eta}$, and get

$$
\left\langle E_{\eta}[F(\eta, \cdot)], \psi\right\rangle=E_{\eta}\left[E_{\omega}[F(\eta, \omega) \cdot \psi(\omega)]\right]=E_{\eta}[\langle F(\eta, \cdot), \psi\rangle]=\left\langle\mathbf{E}_{\eta}[F(\eta, \cdot)], \psi\right\rangle
$$

The next propositions and lemmas deal with the problem of moving the Wick product outside an expectation.

Proposition 10 Let $F: \mathcal{S}^{\prime} \times \mathcal{S}^{\prime} \rightarrow \mathbb{R}$. Assume $G \in(\mathcal{S})^{*}$ and $F(\eta, \cdot) \in L^{2}(\mu)$. In addition, assume that $F(\cdot, \omega) \in L^{1}(\mu), E_{\eta}\left[F^{2}(\eta, \cdot)\right] \in L^{1}(\mu)$, and $F$ and $G$ have chaos expansions

$$
F=\sum_{\alpha} a_{\alpha}(\eta) H_{\alpha}, G=\sum_{\beta} b_{\beta} H_{\beta}
$$

such that

$$
\begin{gathered}
\sum_{\alpha} \alpha ! E_{\eta}\left[\left|a_{\alpha}\right|\right]\left|c_{\alpha}\right|<\infty \\
\sum_{\alpha, \beta}(\alpha+\beta) ! E_{\eta}\left[\left|a_{\alpha}(\eta)\right|\right]\left|b_{\beta}\right|\left|c_{\alpha+\beta}\right|<\infty
\end{gathered}
$$

for all $\psi=\sum_{\alpha} c_{\alpha} H_{\alpha} \in(\mathcal{S})$. Then we have

$$
\mathbb{E}_{\eta}[F(\eta, \omega) \diamond G(\omega)]=\mathbb{E}_{\eta}[F(\eta, \omega)] \diamond G(\omega)
$$


Proof: A direct calculation shows:

$$
\left\langle\mathbf{E}_{\eta}[F(\eta, \omega) \diamond G(\omega)], \psi\right\rangle=E_{\eta}[\langle F \diamond G, \psi\rangle]=E_{\eta}\left[\sum_{\alpha, \beta}(\alpha+\beta) ! a_{\alpha}(\eta) b_{\beta} c_{\alpha+\beta}\right]
$$

Using the assumptions, we can interchange sums and expectation:

$$
=\sum_{\alpha, \beta}(\alpha+\beta) ! E_{\eta}\left[a_{\alpha}(\eta)\right] b_{\beta} c_{\alpha+\beta}=\left\langle\left(\sum_{\alpha} E_{\eta}\left[a_{\alpha}(\eta)\right] H_{\alpha}\right) \diamond G, \psi\right\rangle
$$

which equals, by the assumptions and prop.(9):

$$
=\left\langle E_{\eta}[F(\eta, \cdot)] \diamond G, \psi\right\rangle=\left\langle\mathbf{E}_{\eta}[F(\eta, \cdot)] \diamond G, \psi\right\rangle
$$

The restriction $F \in L^{2}(\mu \times \mu)$ shows up to be sufficient to fulfill the requirements in the proposition above. To prove this, we need some lemmas:

Lemma 11 Assume

$$
\sup _{\alpha} \alpha ! E_{\eta}\left[a_{\alpha}^{2}(\eta)\right]<\infty
$$

Then

$$
X=\sum_{\alpha} E_{\eta}\left[\left|a_{\alpha}(\eta)\right|\right] H_{\alpha}
$$

will be an $(\mathcal{S})^{*}$-element.

Proof: We must show that

$$
\sup _{\alpha} E\left[\mid a_{\alpha}(\eta)\right]^{2} \alpha !(2 \mathbb{N})^{-\alpha q}<\infty
$$

for a $q>0$. By the Hölder inequality, we get

$$
\sup _{\alpha} E\left[\left|a_{\alpha}(\eta)\right|\right]^{2} \alpha !(2 \mathbb{N})^{-\alpha q} \leq \sup _{\alpha} E\left[a_{\alpha}^{2}\right] \alpha !(2 \mathbb{N})^{-\alpha q} \leq \sup _{\alpha} \alpha ! E\left[a_{\alpha}^{2}\right]<\infty
$$

since

$$
(2 \mathbb{N})^{\alpha}=2^{|\alpha|}\left(1^{\alpha_{1}} 2^{\alpha_{2}} \ldots m^{\alpha_{m}}\right) \geq 1, \forall \alpha
$$

Lemma 12 Assume $F(\cdot, \cdot) \in L^{2}(\mu \times \mu)$ and $G \in(\mathcal{S})^{*}$ have chaos expansions

$$
F(\eta, \omega)=\sum_{\alpha} a_{\alpha}(\eta) H_{\alpha}(\omega), G=\sum_{\alpha} b_{\beta} H_{\beta}
$$

Then

$$
\sum_{\alpha} \alpha ! E_{\eta}\left[\left|a_{\alpha}(\eta)\right|\right]\left|c_{\alpha}\right|<\infty
$$

and

$$
\sum_{\alpha, \beta}(\alpha+\beta) ! E_{\eta}\left[\left|a_{\alpha}(\eta)\right|\right]\left|b_{\beta}\right|\left|c_{\alpha+\beta}\right|<\infty
$$

$\forall \psi=\sum_{\alpha} c_{\alpha} H_{\alpha} \in(\mathcal{S})$

Proof: Since $F(\cdot, \cdot) \in L^{2}(\mu \times \mu)$, the Fubini-Tonelli theorem implies

$$
E_{\eta}\left[E_{\omega}\left[F^{2}\right]\right]=E_{\eta}\left[\sum_{\alpha} \alpha ! a_{\alpha}(\eta)^{2}\right]=\sum_{\alpha} \alpha ! E_{\eta}\left[a_{\alpha}(\eta)^{2}\right]<\infty
$$


Hence, by the lemma above

$$
X=\sum_{\alpha} E_{\eta}\left[\left|a_{\alpha}(\eta)\right|\right] H_{\alpha} \in(\mathcal{S})^{*}
$$

This gives

$$
\infty>\langle X \diamond \bar{G}, \bar{\psi}\rangle=\sum_{\alpha, \beta}(\alpha+\beta) ! E_{\eta}\left[\mid a_{\alpha}(\eta)\right]\left|b_{\beta}\right|\left|c_{\alpha+\beta}\right|
$$

where

$$
\bar{\psi}=\sum_{\alpha}\left|c_{\alpha}\right| H_{\alpha} \in(\mathcal{S})
$$

and

$$
\bar{G}=\sum_{\beta}\left|b_{\beta}\right| H_{\beta} \in(\mathcal{S})^{*}
$$

In addition

$$
\infty\rangle\langle X, \bar{\psi}\rangle=\sum_{\alpha} \alpha ! E_{\eta}\left[\left|a_{\alpha}(\eta)\right|\right]\left|c_{\alpha}\right|
$$

Proposition 13 Assume $F(\cdot, \cdot) \in L^{2}(\mu \times \mu)$ and $G \in(\mathcal{S})^{*}$, then

$$
\mathbb{E}_{\eta}[F(\eta, \omega) \diamond G(\omega)]=\mathbb{E}_{\eta}[F(\eta, \omega)] \diamond G(\omega)
$$

Proof: By lemma (12) and the Fubini-Tonelli theorem, the assumptions in prop.(10) are fullfiled. Hence, the proposition follows.

We now move on with the problem of showing that

$$
\operatorname{Exp} W_{\gamma(\eta)}(\omega) \diamond W_{\psi}(\omega) \in L^{2}(\mu)
$$

as a function of $\eta$. The Wick product is taken w.r.t. $\omega$. The rest of this section is dedicated to this problem. We start with stating a preliminary result.

Proposition 14 Assume $Y \in L^{2}(\mu)$ and $\psi \in C_{c}^{\infty}$. If $\psi(s) Y(\omega)$ is Skorohod integrable, then

$$
Y(\omega) \diamond W_{\psi}(\omega)=\int_{a}^{b} \psi(s) Y(\omega) \delta B_{s}(\omega)
$$

where $\operatorname{supp}(\psi) \subset[a, b]$.

Proof: Let $Y$ have the chaos expansion

$$
Y(\omega)=\sum_{\alpha} c_{\alpha} H_{\alpha}(\omega)
$$

By (16), we have

$$
W_{\psi}(\omega)=\sum_{k}\left(\psi, \xi_{k}\right) H_{\epsilon_{k}}(\omega)
$$

Results from [1] imply

$$
\begin{gathered}
Y(\omega) \diamond W_{\psi}(\omega)=\sum_{k, \alpha} c_{\alpha}\left(\psi, \xi_{k}\right) H_{\alpha+\epsilon_{k}}=\sum_{k, \alpha}\left(\psi c_{\alpha}, \xi_{k}\right) H_{\alpha+\epsilon_{k}} \\
=\sum_{k, \alpha}\left(\int_{a}^{b} \psi(s) c_{\alpha} \xi_{k}(s) d s\right) H_{\alpha+\epsilon_{k}}=\int_{a}^{b} \psi(s) Y(\omega) \diamond W_{s}(\omega) d s=\int_{a}^{b} \psi(s) Y(\omega) \delta B_{s}(\omega)
\end{gathered}
$$

We state the result which sums up what we need for the theorem in section 5: 
Proposition 15 Given $F(\cdot, \cdot) \in L^{2}(\mu \times \mu)$ by

$$
F(\eta, \omega)=\operatorname{Exp}_{\gamma(\eta)}(\omega)
$$

where $\gamma(\eta, s)$ is bounded in both variables. Then

$$
F(\cdot, \omega) \diamond W_{\psi}(\omega) \in L^{2}(\mu)
$$

as a function of $\eta$, where the Wick product is taken w.r.t. $\omega$.

Proof: By the Fubini-Tonelli theorem, it is sufficient to show that

$$
F(\eta, \omega) \diamond W_{\psi}(\omega) \in L^{2}(\mu \times \mu)
$$

Using Prop.(3.1) in [10], we obtain

$$
\begin{gathered}
E_{\eta, \omega}\left[\left(F(\eta, \omega) \diamond W_{\psi}(\omega)\right)^{2}\right]=E_{\eta, \omega}\left[\left(\int_{a}^{b} \psi(s) F(\eta, \omega) \delta B_{s}(\omega)\right)^{2}\right] \\
=E_{\eta}\left[E_{\omega}\left[\left(\int_{a}^{b} \psi(s) F(\eta, \omega) \delta B_{s}(\omega)\right)^{2}\right]\right] \leq E_{\eta}\left[\int_{a}^{b} \psi^{2}(s) E_{\omega}\left[\left(E_{x p} W_{\gamma(\eta)}\right)^{2}+\left(N E x p W_{\gamma(\eta)}\right)^{2}\right] d s\right] \\
=\|\psi\|^{2} E_{\eta}\left[E_{\omega}\left[\left(E x p W_{\gamma(\eta)}\right)^{2}+\left(N E x p W_{\gamma(\eta)}\right)^{2}\right]\right]<\infty
\end{gathered}
$$

where $N$ is the number operator, also called the Ohrnstein-Uhlenbeck operator.

\section{The Solution Candidate.}

We will show how the Hermite transform can be used to produce an explicit solution candidate to problem (1) and (2). Moreover, we use the singular white noise $W_{s}$ to construct a candidate (see (40)). However, since $W_{s}$ is a very complicated element to handle, we have not been able to prove that the candidate (40) is the solution of (1) and (2) in a strong sense. We have only been able to prove the result for the weaker solution concept involving functional processes. In the second part of this section, we study some properties of the solution candidate.

We start with formulating a slight modification of th.(5.7.6) from [9], where a stochastic representation of the solution of a deterministic Cauchy problem is given. In the formulation, let $L$ be the generator to the Ito diffusion $X_{s}$, and $E^{t, x}$ the expectation taken under the condition that $X_{s}=X_{s}^{t, x}, s \geq t$.

Theorem 16 Assume $k:[0, T] \times \mathbb{R} \rightarrow \mathbb{R}$ continuous and bounded, $p_{0}: \mathbb{R} \rightarrow \mathbb{R}$ continuous and positive. Let $u:[0, T] \times \mathbb{R} \rightarrow \mathbb{R}$ be of class $C^{1,2}([0, T) \times \mathbb{R})$ and satisfy the Cauchy problem

$$
\begin{aligned}
& \frac{\partial u}{\partial t}+k u=L u \\
& u(0, x)=p_{0}(x)
\end{aligned}
$$

as well as the polynomial growth condition

$$
\max _{0 \leq t \leq T}|u(t, x)| \leq M\left(1+|x|^{2 \mu}\right)
$$

for some $M>0, \mu \geq 1$. Then $u(t, x)$ admits the stochastic representation

$$
u(t, x)=E^{T-t, x}\left[p_{0}\left(X_{T}\right) \exp \left\{-\int_{T-t}^{T} k\left(T-s, X_{s}\right) d s\right\}\right]
$$

on $[0, T] \times \mathbb{R}$. In particular, such a solution is unique. 
To translate our stochastic partial differential equation into a deterministic problem, we must write (1) and (2) in integral form:

$$
\phi_{t}(x, \omega)=p_{0}(x)+\int_{0}^{t} L \phi_{s}(x, \omega)-c(s, x) \phi_{s}(x, \omega) d s+\int_{0}^{t} h(s, x) \phi_{s}(x, \omega) d B_{s}
$$

Assuming $\phi_{s}(x, \omega)$ is Ito integrable, results from [1] imply

$$
\int_{0}^{t} h(s, x) \phi_{s}(x, \omega) d B_{s}=\int_{0}^{t} h(s, x) \phi_{s}(x, \omega) \diamond W_{s}(\omega) d s
$$

Differentiation w.r.t. to $t$ gives the problem:

$$
\begin{gathered}
\frac{\partial \phi_{t}}{\partial t}(x, \omega)=L \phi_{t}(x, \omega)-c(t, x) \phi_{t}(x, \omega)+h(t, x) \phi_{t}(x, \omega) \diamond W_{t}(\omega) \\
\phi_{0}(x, \omega)=p_{0}(x)
\end{gathered}
$$

Invoking the Hermite transform, we get

$$
\begin{gathered}
\frac{\partial \tilde{\phi}_{t}(x, z)}{\partial t}=L \tilde{\phi}_{t}(x, z)-\left(c(t, x)-h(t, x) \tilde{W}_{t}(z)\right) \tilde{\phi}_{t}(x, z) \\
\tilde{\phi}_{0}(x, z)=p_{0}(x)
\end{gathered}
$$

where

$$
\tilde{W}_{t}(z)=\sum_{k=1}^{\infty} \xi_{k}(t) z_{k}
$$

$\left\{\xi_{k}\right\}_{k=1}^{\infty}$ is the usual orthonormal basis in $L^{2}(\mathbb{R})$, and $z \in \mathbb{C}_{0}^{\mathbb{N}}$. Hence, we have translated our original problem to a deterministic one with parameters $z_{k}$. Invoking theorem(16) on the problem (38) and (39), a solution will have the representation:

$$
\begin{aligned}
\tilde{\phi}_{t}(x, z)= & E_{\eta}^{T-t, x}\left[p_{0}\left(X_{T}(\eta)\right) \exp \left(-\int_{T-t}^{T} c\left(T-s, X_{s}(\eta)\right) d s\right) \times\right. \\
& \left.\exp \left(\int_{T-t}^{T} h\left(T-s, X_{s}(\eta)\right) \tilde{W}_{T-s}(z) d s\right)\right]
\end{aligned}
$$

From $[4$, p.28] we have

$$
\mathcal{H}\left(\operatorname{Exp} W_{\rho}\right)=\exp \left(\mathcal{H} W_{\rho}\right)
$$

where $\mathcal{H}$ is the Hermite transform, and $E_{x p} W_{\rho}$ is the Wick exponential. Hence

$$
\begin{gathered}
\phi_{t}(x, \omega)=E^{T-t, x}\left[p_{0}\left(X_{T}(\eta)\right) \exp \left(-\int_{T-t}^{T} c\left(T-s, X_{s}(\eta)\right) d s\right) \times\right. \\
\left.\operatorname{Exp}\left(\int_{T-t}^{T} h\left(T-s, X_{s}(\eta)\right) \diamond W_{T-s}(\omega) d s\right)\right]
\end{gathered}
$$

A change of variables gives

$$
\phi_{t}(x, \omega)=E^{T-t, x}\left[p_{0}\left(X_{T}\right) \exp \left(-\int_{0}^{t} c\left(s, X_{T-s}\right) d s\right) \operatorname{Exp}\left(\int_{0}^{t} h\left(s, X_{T-s}\right) \diamond W_{s}(\omega) d s\right)\right]
$$

$E^{T-t, x}$ is the expectation taken w.r.t. the measure induced by the Ito diffusion $X_{s}=X_{s}^{T-t, x}, s \geq$ $T-t$. Note that the Wick product turns into an ordinary product, since $h$ does not depend on $\omega$. Hence

$$
E^{T-t, x}\left[p_{0}\left(X_{T}\right) \exp \left(-\int_{0}^{t} c\left(s, X_{T-s}\right) d s\right) \operatorname{Exp}\left(\int_{0}^{t} h\left(s, X_{T-s}\right) W_{s}(\omega) d s\right)\right]
$$




$$
=E^{0, x}\left[p_{0}\left(X_{T-(T-t)}\right) \exp \left(-\int_{0}^{t} c\left(s, X_{T-(T-t)-s}\right) d s\right) \operatorname{Exp}\left(\int_{0}^{t} h\left(s, X_{T-(T-t)-s}\right) W_{s}(\omega) d s\right)\right]
$$

which gives the formula

$$
\phi_{t}(x, \omega)=E^{x}\left[p_{0}\left(X_{t}\right) \exp \left(-\int_{0}^{t} c\left(s, X_{t-s}\right) d s\right) \operatorname{Exp}\left(\int_{0}^{t} h\left(s, X_{t-s}\right) W_{s}(\omega) d s\right)\right]
$$

In [2], it is proved that $(40)$ solves $(1)$ and $(2)$ in an $(\mathcal{S})^{*}$-sense. However, the solution concept does not have any physical implications, and it can not be used in connection with the filtering problem. The solution concept in $(\mathcal{S})^{*}$ was invoked to cope with the difficulties caused by the singular white noise $W_{t}$. As far as the author can see, the methods used in the present paper are not adoptable to the problem of showing that (40) solves (1) and (2) in a strong $L^{2}(\mu)$ sense.

Inspired by the above derivation, we will show that

$$
\phi_{t}^{\psi}(x, \omega)=E^{x}\left[p_{0}\left(X_{t}\right) \exp \left(-\int_{0}^{t} c\left(t-s, X_{s}\right) d s\right) \operatorname{Exp}\left(\int_{0}^{t} h\left(s, X_{t-s}\right) W_{\psi_{s}}(\omega) d s\right)\right]
$$

is a functional process solution, where $\psi_{t}(x)=\psi(x-t)$ for $\psi \in C_{c}^{\infty}$.

After this demonstration of the Hermite transformation technique, let us consider some properties of our solution candidate (41). First of all we will show that $\phi_{t}^{\psi}$ is an $L^{2}$-process: Consider the integral

Put

$$
\int_{0}^{t} h\left(s, X_{t-s}\right) W_{\psi_{s}}(\omega) d s
$$

$$
f_{\eta}(s)=\mathcal{X}_{[0, t]}(s) h\left(s, X_{t-s}(\eta)\right)
$$

By lemma (3.3) in [15] we get the following:

$$
\int_{0}^{t} h\left(s, X_{t-s}\right) W_{\psi_{s}}(\omega) d s=\int_{\mathbb{R}} f_{\eta}(s) W_{\psi_{s}}(\omega) d s=\int_{\mathbb{R}}\left(\psi * f_{\eta}\right)_{s} \delta B_{s}
$$

where the last integral is the Skorohod integral and $*$ is the convolution product defined as

$$
\left(\psi * f_{\eta}\right)_{s}=\int_{\mathbb{R}} \psi(s-u) f_{\eta}(u) d u
$$

If we assume that $h$ is bounded, we get $f_{\eta} \in L^{2}(d s)$ and, by Youngs inequality ([3, (8.7)]),

$$
\left(\psi * f_{\eta}\right)_{s} \in L^{2}(d s) \forall \psi \in C_{c}^{\infty}
$$

For each $\eta,\left(\psi * f_{\eta}\right)_{s}$ is a deterministic function in $L^{2}$. Therefore

$$
\int_{0}^{t} h\left(s, X_{t-s}(\eta)\right) W_{\psi_{s}}(\omega) d s=\int_{\mathbb{B}}\left(\psi * f_{\eta}\right)_{s} d B_{s}(\omega)=W_{\gamma(\eta)}(\omega)
$$

where

$$
\gamma(\eta, s)=\left(\psi * f_{\eta}\right)_{s}
$$

Define

$$
F(\eta, \omega)=p_{0}\left(X_{t}(\eta)\right) \exp \left(-\int_{0}^{t} c\left(s, X_{s}(\eta)\right) d s\right) \operatorname{Exp}\left(W_{\gamma(\eta)}(\omega)\right)
$$

We have the following easy, but important result:

Proposition 17 Assume $p_{0}, c$ and $h$ are bounded functions. Then

$$
F(\cdot, \cdot) \in L^{2}(\mu \times \mu)
$$

and, $\forall \psi \in C_{c}^{\infty}$

$$
\phi_{t}^{\psi}(x, .) \in L^{2}(\mu)
$$


Proof: We use the boundedness of $h$ and the Fubini-Tonelli theorem:

$$
E_{\eta, \omega}\left[F^{2}\right]=E_{\eta}\left[E_{\omega}\left[F^{2}\right]\right]=E_{\eta}\left[p_{0}^{2}\left(X_{t}\right) \exp \left(-2 \int_{0}^{t} c\left(s, X_{s}\right) d s\right) \cdot E_{\omega}\left[\left(\operatorname{Exp} W_{\gamma(\eta)}(\omega)\right)^{2}\right]\right]<\infty
$$

The Hőlder inequality together with the Fubini-Tonelli theorem gives the latter result.

Hence, our solution candidate is a functional process. In order to prove that our $\phi_{t}^{\psi}$ satisfies (28), i.e. that it satisfies the Cauchy problem

$$
\begin{gathered}
\frac{\partial \phi_{t}^{\psi}}{\partial t}=L \phi_{t}^{\psi}-\left(c(t, x)-h(t, x) W_{\psi_{t}}(\omega)\right) \diamond \phi_{t}^{\psi} \\
\phi_{0}^{\psi}(x, \omega)=p_{0}(x)
\end{gathered}
$$

$\phi_{t}^{\psi}$ has to be $t$-differentiable. To accomplish this, we need the following technical result:

Lemma 18 Suppose $\psi \in C_{c}^{\infty}$ and $h, \frac{\partial h}{\partial t}$ are bounded functions. Set

$$
g_{\eta}(t, s, u)=\psi(s-(t-u)) h\left(t-u, X_{u}(\eta)\right)
$$

Then there exists a $q \in L^{1}(d s)$ such that

$$
\left|\frac{\partial}{\partial t}\left(\int_{0}^{t} g_{\eta}(t, s, u) d u\right)^{2}\right| \leq q(s)
$$

Proof: We show this by direct calculation:

$$
\begin{gathered}
\left|\frac{\partial}{\partial t}\left(\int_{0}^{t} g_{\eta}(t, s, u) d u\right)^{2}\right|=\left|2\left\{g_{\eta}(t, s, t)+\int_{0}^{t} \frac{\partial g_{\eta}}{\partial t}(t, s, u) d u\right\}\left(\int_{0}^{t} g_{\eta}(t, s, u) d u\right)\right| \\
=2 \mid\left\{\psi(s) h\left(0, X_{t}\right)+\int_{0}^{t} \frac{\partial \psi}{\partial t}(s-(t-u)) h\left(t-u, X_{u}\right) d u+\int_{0}^{t} \psi(s-(t-u)) \frac{\partial h}{\partial t}\left(t-u, X_{u}\right) d u\right\} \times \\
\quad\left(\int_{0}^{t} \psi(s-(t-u)) h\left(t-u, X_{u}\right) d u\right) \mid \\
\leq 2\left\{\left|\psi(s) h\left(0, X_{t}\right)\right|+\int_{0}^{t}\left|\frac{\partial \psi}{\partial t}(s-(t-u)) h\left(t-u, X_{u}\right)\right| d u+\int_{0}^{t}\left|\psi(s-(t-u)) \frac{\partial h}{\partial t}\left(t-u, X_{u}\right)\right| d u\right\} \times \\
\left(\int_{0}^{t}\left|\psi(s-(t-u)) h\left(t-u, X_{u}\right)\right| d u\right)
\end{gathered}
$$

By restrictions on $h$ :

$$
\begin{gathered}
\leq 2\left\{C_{1}|\psi(s)|+C_{1} \int_{0}^{t}\left|\psi^{\prime}(s-(t-u))\right| d u+C_{2} \int_{0}^{t}|\psi(s-(t-u))| d u\right\} \times \\
C_{1} \int_{0}^{t}|\psi(s-(t-u))| d u
\end{gathered}
$$

Recall that $t \in[0, T]$. Consider each term seperately:

$$
|\psi(s)| \int_{0}^{t}|\psi(s-(t-u))| d u \leq A_{1} T|\psi(s)| \in L^{1}(d s)
$$

since $\psi \in C_{c}^{\infty}$, and hence bounded and in $L^{1}$. By t-continuity of the integral, and that $\psi^{\prime} \in C_{c}^{\infty}$, we get, for a $t_{0} \in[0, T]$

$$
\int_{0}^{t}\left|\psi^{\prime}(s-(t-u))\right| d u \int_{0}^{t}|\psi(s-(t-u))| d u \leq A_{2} T \int_{0}^{t}|\psi(s-(t-u))| d u
$$




$$
\leq A_{2} T \int_{0}^{t_{0}} \mid \psi\left(s-\left(t_{0}-u\right)\right) d u
$$

The Tonelli theorem implies that

$$
A_{2} T \int_{0}^{t_{0}} \mid \psi\left(s-\left(t_{0}-u\right)\right) d u \in L^{1}(d s)
$$

since

$$
\int_{\mathbb{B}}\left(\int_{0}^{t_{0}}|\psi(s-(t-u))| d u\right) d s=\int_{0}^{t_{0}}\|\psi\|_{L^{1}} d u=t_{0}\|\psi\|_{L^{1}}<\infty
$$

The same argument gives

$$
\int_{0}^{t}|\psi(s-(t-u))| d u \int_{0}^{t}|\psi(s-(t-u))| d u \leq A_{1} T \int_{0}^{t_{0}}\left|\psi\left(s-\left(t_{0}-u\right)\right)\right| d u \in L^{1}(d s)
$$

Hence, the lemma follows.

Proposition 19 Assume $p_{0} \in C_{0}^{2}$ and $c, \frac{\partial c}{\partial t}, h, \frac{\partial h}{\partial t}$ bounded. Then $\phi_{t}^{\psi}$ is t-differentiable.

Proof: Let

$$
f_{\eta}(s)=\mathcal{X}_{[0, t]}(s) h\left(s, X_{t-s}(\eta)\right)
$$

and, as before,

$$
\gamma(\eta, s)=\left(\psi * f_{\eta}\right)_{s}
$$

Then

$$
\begin{gathered}
\operatorname{Exp}\left(\int_{0}^{t} h\left(s, X_{t-s}\right) W_{\psi_{s}} d s\right)=\operatorname{Exp}\left(W_{\gamma}(\omega)\right)=\exp \left(\int \gamma(s) d B_{s}-1 / 2\|\gamma\|^{2}\right) \\
=\exp \left(\int_{\mathbb{I}}\left(\psi * f_{\eta}\right)_{s} d B_{s}-1 / 2 \int_{\mathbb{R}}\left(\psi * f_{\eta}\right)_{s}^{2} d s\right) \\
=\exp \left(\int_{0}^{t} h\left(s, X_{t-s}\right) W_{\psi_{s}} d s\right) \cdot \exp \left(-1 / 2 \int_{\mathbb{B}}\left(\int_{0}^{t} \psi(s-u) h\left(u, X_{t-u}\right) d u\right)^{2} d s\right)
\end{gathered}
$$

Under change of variables, this will be equal to

$$
=\exp \left(\int_{0}^{t} h\left(t-s, X_{s}\right) W_{\psi_{t-s}} d s\right) \cdot \exp \left(-1 / 2 \int_{\mathbb{B}}\left(\int_{0}^{t} \psi(s-(t-u)) h\left(t-u, X_{u}\right) d u\right)^{2} d s\right)
$$

Now define $A_{t}=p_{0}\left(X_{t}\right), R_{t}=\exp \left(-\int_{0}^{t} c\left(t-s, X_{s}\right) d s\right), S_{t}=\exp \left(\int_{0}^{t} h\left(t-s, X_{s}\right) W_{\psi_{t-s}}(\omega) d s\right)$ and $V_{t}=\exp \left(-1 / 2 \int_{\mathbb{R}}\left(\int_{0}^{t} g_{\eta}(t, s, u) d u\right)^{2} d s\right)$, where $g_{\eta}(t, s, u)=\psi(s-(t-u)) h\left(t-u, X_{u}\right)$. The idea now is to write these processes as stochastic integrals, and use the Ito-formula and lemma(7.8) in [14]. By (7.20) in [14], $A_{t}$ can be written as a stochastic integral. The conditions on $c$ give the same result for $R_{t}$, by a direct differentiation w.r.t. $t$. $S_{t}$ and $V_{t}$ need a more careful examination:

The conditions on $h$, and the result $\frac{\partial}{\partial t} W_{\psi_{t-s}}(\omega)=-W_{\psi_{t-s}^{\prime}}(\omega)$, justify the following direct calculation:

$$
\frac{d S_{t}}{d t}=\exp \left(\int_{0}^{t} h\left(t-s, X_{s}\right) W_{\psi_{t-s}} d s\right)\left(h\left(0, X_{t}\right) W_{\psi_{0}}+\int_{0}^{t} \frac{\partial}{\partial t}\left(h\left(t-s, X_{s}\right) W_{\psi_{t-s}}\right) d s\right)
$$

which implies

$$
d S_{t}=S_{t} \cdot\left\{h\left(0, X_{t}\right) W_{\psi_{0}}+\int_{0}^{t}\left(\frac{\partial h}{\partial t}\left(t-s, X_{s}\right) W_{\psi_{t-s}}-h\left(t-s, X_{s}\right) W_{\psi_{t-s}^{\prime}}\right) d s\right\} d t
$$


Since $\psi \in \mathcal{S}$ and $h, \frac{\partial h}{\partial t}$ are bounded, the lemma above gives

$$
\left|\frac{\partial}{\partial t}\left(\int_{0}^{t} g_{\eta}(t, s, u) d u\right)^{2}\right| \leq q(s) \in L^{1}(d s)
$$

Th.(2.27) in [3] then implies:

$$
\frac{\partial}{\partial t} \int_{\mathbb{R}}\left(\int_{0}^{t} g_{\eta}(t, s, u) d u\right)^{2} d s=\int_{\mathbb{B}}\left(\frac{\partial}{\partial t}\left(\int_{0}^{t} g_{\eta}(t, s, u) d u\right)^{2}\right) d s
$$

and hence

$$
\begin{gathered}
\frac{d V_{t}}{d t}=\exp \left(-1 / 2 \int_{\mathbb{R}}\left(\int_{0}^{t} g_{\eta}(t, s, u) d u\right)^{2} d s\right) \cdot \frac{\partial}{\partial t}\left(-1 / 2 \int_{\mathbb{R}}\left(\int_{0}^{t} g_{\eta}(t, s, u) d u\right)^{2} d s\right) \\
=\exp \left(-1 / 2 \int_{\mathbb{B}}\left(\int_{0}^{t} g_{\eta}(t, s, u) d u\right)^{2} d s\right) \cdot\left(-1 / 2 \int_{\mathbb{B}}\left(\frac{\partial}{\partial t}\left(\int_{0}^{t} g_{\eta}(t, s, u) d u\right)^{2}\right) d s\right) \\
=\exp \left(-1 / 2 \int_{\mathbb{R}}\left(\int_{0}^{t} g_{\eta}(t, s, u) d u\right)^{2} d s\right)\left(-1 / 2 \int_{\mathbb{R}} 2\left(\int_{0}^{t} g_{\eta}(t, s, u) d u\right)\left(g_{\eta}(t, s, t)+\int_{0}^{t} \frac{\partial g_{\eta}}{\partial t}(t, s, u) d u\right) d s\right)
\end{gathered}
$$

Hence

$$
d V_{t}=V_{t} \cdot\left(\int_{\mathbb{R}}\left\{\left(\int_{0}^{t} g_{\eta}(t, s, u) d u\right)\left(g_{\eta}(t, s, t)+\int_{0}^{t} \frac{\partial g_{\eta}}{\partial t}(t, s, u) d u\right)\right\} d s\right) d t
$$

All 4 processes can then be written as stochastic integrals, and lemma(7.8) in [14] gives the differentiability.

\section{The Functional Process Solution.}

We are now in the position to prove the main result of this paper:

Theorem 20 Assume $p_{0} \in C_{0}^{2}$ and $c, h, \frac{\partial c}{\partial t}, \frac{\partial h}{\partial t}$ are bounded. In addition, assume that $c(t, \cdot), h(t, \cdot)$ are continuous. Then

$$
\phi_{t}^{\psi}(x, \omega)=E_{\eta}^{x}\left[p_{0}\left(X_{t}\right) \exp \left(-\int_{0}^{t} c\left(t-s, X_{s}\right) d s\right) \operatorname{Exp}\left(\int_{0}^{t} h\left(s, X_{t-s}\right) W_{\psi_{s}}(\omega) d s\right)\right]
$$

is an $L^{2}$-functional process solution of the stochastic Cauchy problem (1) and (2).

Proof: We prove that $\phi_{t}^{\psi}$ satisfies (28). A change of variables gives

$$
\int_{0}^{t} h\left(s, X_{t-s}\right) W_{\psi_{s}} d s=\int_{0}^{t} h\left(t-s, X_{s}\right) W_{\psi_{t-s}} d s
$$

Now

$$
\frac{1}{u}\left(E^{x}\left[\phi_{t}\left(X_{u}\right)\right]-\phi_{t}(x)\right)=\frac{1}{u}\left(E^{x}\left[E^{X_{u}}\left[p_{0}(.) \exp (.) \operatorname{Exp}(.)\right]\right]-\phi_{t}(x)\right)
$$

The Markov Property implies

$$
\frac{1}{u}\left(E^{x}\left[p_{0}\left(X_{t+u}\right) \exp \left(-\int_{0}^{t} c\left(t-s, X_{s+u}\right) d s\right) \operatorname{Exp}\left(\int_{0}^{t} h\left(t-s, X_{s+u}\right) W_{\psi_{t-}} d s\right)\right]-\phi_{t}(x)\right)
$$

A change of variables

$$
\frac{1}{u}\left(E ^ { x } \left[p_{0}\left(X_{t+u}\right) \exp \left(-\int_{u}^{t+u} c\left(t+u-s, X_{s}\right) d s\right) \times\right.\right.
$$




$$
\begin{gathered}
\left.\left.\operatorname{Exp}\left(\int_{u}^{t+u} h\left(t+u-s, X_{s}\right) W_{\psi_{t+u-s}} d s\right)\right]-\phi_{t}(x)\right) \\
=\frac{1}{u}\left(E ^ { x } \left[p_{0}\left(X_{t+u}\right) \exp \left(-\int_{0}^{t+u} c\left(t+u-s, X_{s}\right) d s\right) \operatorname{Exp}\left(\int_{0}^{t+u} h\left(t+u-s, X_{s}\right) W_{\psi_{t+u-s}} d s\right) \times\right.\right. \\
\left.\left.\exp \left(\int_{0}^{u} c\left(t+u-s, X_{s}\right) d s\right) \diamond \operatorname{Exp}\left(-\int_{0}^{u} h\left(t+u-s, X_{s}\right) W_{\psi_{t+u-s}} d s\right)\right]-\phi_{t}(x)\right) \\
=\frac{1}{u}\left(E ^ { x } \left[p_{0}\left(X_{t+u}\right) \exp \left(-\int_{0}^{t+u} c\left(t+u-s, X_{s}\right) d s\right) \times\right.\right. \\
\left.\left.E x p\left(\int_{0}^{t+u} h\left(t+u-s, X_{s}\right) W_{\psi_{t+u-s}} d s\right)\right]-\phi_{t}(x)\right) \\
+\frac{1}{u} E^{x}\left[p_{0}\left(X_{t+u}\right) \exp \left(-\int_{0}^{t+u} c\left(t+u-s, X_{s}\right) d s\right) \operatorname{Exp}\left(\int_{0}^{t+u} h\left(t+u-s, X_{s}\right) W_{\psi_{t+u-s}} d s\right) \diamond\right. \\
\left.\left\{\exp \left(\int_{0}^{u} c\left(t+u-s, X_{s}\right) d s\right) \operatorname{Exp}\left(-\int_{0}^{u} h\left(t+u-s, X_{s}\right) W_{\psi_{t+u-s}} d s\right)-1\right\}\right]
\end{gathered}
$$

Considering the first term we see that this can be written on compact form as

$$
\frac{1}{u}\left(\phi_{t+u}(x)-\phi_{t}(x)\right)
$$

By prop.(19) above, this converges to $\frac{\partial \phi_{t}}{\partial t}(x)$ when $u \downarrow 0$.

The second term can be written as

$$
\begin{gathered}
E^{x}\left[p_{0}\left(X_{t+u}\right) \exp \left(-\int_{0}^{t+u} c\left(t+u-s, X_{s}\right) d s\right) \operatorname{Exp}\left(\int_{0}^{t+u} h\left(t+u-s, X_{s}\right) W_{\psi_{t+u-s}} d s\right) \diamond\right. \\
\left.\frac{1}{u}\left\{\exp \left(\int_{0}^{u} c\left(t+u-s, X_{s}\right) d s\right) E x p\left(-\int_{0}^{u} h\left(t+u-s, X_{s}\right) W_{\psi_{t+u-s}} d s\right)-1\right\}\right]
\end{gathered}
$$

which we claim converges to

$$
\phi_{t}(x, \omega) \diamond\left(c(t, x)-h(t, x) W_{\psi_{t}}(\omega)\right)
$$

when $u \downarrow 0$ :

Define

$$
G(u)=\exp \left(\int_{0}^{u} c\left(t+u-s, X_{s}\right) d s\right) E x p\left(-\int_{0}^{u} h\left(t+u-s, X_{s}\right) W_{\psi_{t+u-s}} d s\right)
$$

Then

$$
\begin{gathered}
\lim _{u \downarrow 0} \frac{1}{u}(\exp (\ldots) \operatorname{Exp}(\ldots)-1)=\lim _{u \downarrow 0} \frac{1}{u}(G(u)-G(0))=\left.\left(\frac{d}{d u} G(u)\right)\right|_{u=0} \\
=\left(G ( u ) \diamond \left\{c\left(t, X_{u}\right)+\int_{0}^{u} \frac{\partial}{\partial t}\left(c\left(t+u-s, X_{s}\right)\right) d s-\left(h\left(t, X_{u}\right) W_{\psi_{t}}+\right.\right.\right. \\
\left.\left.\left.\int_{0}^{u} \frac{\partial}{\partial u}\left(h\left(t+u-s, X_{s}\right) W_{\psi_{t+u-s}}\right) d s\right)\right\}\right)\left.\right|_{u=0} \\
=c(t, x)-h(t, x) W_{\psi_{t}}(\omega)
\end{gathered}
$$

Hence, (46) converges to

$E^{x}\left[p_{0}\left(X_{t}\right) \exp \left(-\int_{0}^{t} c\left(t-s, X_{s}\right) d s\right) \operatorname{Exp}\left(\int_{0}^{t} h\left(t-s, X_{s}\right) W_{\psi_{t-s}}(\omega) d s\right) \diamond\left(c(t, x)-h(t, x) W_{\psi_{t}}(\omega)\right)\right]$ 
Prop.(15) ensures that the integrand in this expectation is an element of $L^{2}(\mu)$ as a function of $\eta$. Invoking prop.(17) in prop.(13), we can move the Wick product outside the expectation, $E_{\eta}^{x}$, and hence the claim follows.

Since

$$
L \phi_{t}(x)=\lim _{u \downarrow 0} \frac{1}{u}\left(E^{x}\left[\phi_{t}\left(X_{u}\right)\right]-\phi_{t}(x)\right)
$$

the proof is complete.

We end this paper by looking at the connection to filtering theory. Consider the system process

$$
d Z_{t}=b\left(Z_{t}\right) d t+\sigma\left(Z_{t}\right) d U_{t}
$$

where $U_{t}$ is a Brownian motion. The observation process is given by

$$
d Y_{t}=h\left(t, Z_{t}\right) d t+d V_{t}
$$

where $V_{t}$ is a Brownian motion independent of $U_{t}$. For this filtering problem, the Wong-Zakai equation is

$$
\begin{gathered}
d \phi_{t}(x, \omega)=A_{x}^{*} \phi_{t}(x, \omega)+h(t, x) \phi_{t}(x, \omega) d Y_{t}(\omega) \\
\phi_{0}(x, \omega)=p_{0}(x)
\end{gathered}
$$

In the context of filtering theory, $Y_{t}$ is a Brownian motion. $A_{x}^{*}$ is the adjoint operator to the infinitesimal generator $A_{x}$ associated to the system process $Z_{t}$. $A_{x}$ considered as a differential operator equals (in one-dimensional space)

$$
A_{x}=b(x) \frac{d}{d x}+\frac{1}{2} \sigma^{2}(x) \frac{d^{2}}{d x^{2}}
$$

Under appropriate differential hypotheses on $b, \sigma$ this yields

$$
A_{x}^{*}=\left(\rho(x) \frac{d}{d x}+\frac{1}{2} \sigma^{2}(x) \frac{d^{2}}{d x^{2}}\right)-c(x)=L_{x}-c(x)
$$

where

$$
c(x)=b^{\prime}(x)-\left(\sigma^{\prime}(x)\right)^{2}-\sigma(x) \sigma^{\prime \prime}(x)
$$

and

$$
\rho(x)=2 \sigma(x) \sigma^{\prime}(x)-b(x)
$$

Hence, (49) and (50) can be formulated

$$
\phi_{t}(x, \omega)=p_{0}(x)+\int_{0}^{t}\left\{L_{x} \phi_{s}(x, \omega)-c(x) \phi_{s}(x, \omega)\right\} d s+h(x) \int_{0}^{t} \phi_{s}(x, \omega) d Y_{s}(\omega)
$$

The functional process version of this equation is (28), with $c$ and $h$ as above.

For the special case of nonlinear observation of Brownian motion, i.e. $Z_{t}=U_{t}$, we have

$$
L_{x}=\frac{1}{2} \triangle_{x}
$$

since $b=0$ and $\sigma=1$. Of course, $c=0$. Then

$$
\phi_{t}^{\psi}(x, \omega)=E^{x}\left[p_{0}\left(B_{t}\right) \operatorname{Exp}\left(\int_{0}^{t} h\left(s, B_{t-s}\right) W_{\psi_{s}}(\omega) d s\right)\right]
$$

is a functional process solution of the Wong-Zakai equation

$$
\phi_{t}(x, \omega)=p_{0}(x)+\frac{1}{2} \int_{0}^{t} \triangle_{x} \phi_{s}(x, \omega) d s+\int_{0}^{t} h(s, x) \phi_{s}(x, \omega) d Y_{s}(\omega)
$$


Note that the stochastic partial differential equation (1) and (2) also can be considered as a noisy heat transfer model. For the case $h=1, c=0$ and $L_{x}=\frac{1}{2} \triangle$ the solution takes the nice form

$$
\phi_{t}^{\psi}(x, \omega)=E^{x}\left[p_{0}\left(B_{t}\right)\right] \cdot \operatorname{Exp}\left(\int_{0}^{t} W_{\psi_{s}}(\omega) d s\right)
$$

Acknowledgements: Parts of this paper is taken from the author's cand.scient thesis [2]. The author would like to thank professor Bernt $\varnothing$ ksendal for his helpful suggestions and for many inspiring and fruitful discussions during the work with this paper. The author would also like to thank the Norwegian Research Council (NAVF; 17.515/1017) for their financial support.

\section{References}

[1] F. E. Benth: Integrals In The Hida Distribution Space $(\mathcal{S})^{*}$. To appear in Stochastic Analysis and Related Topics, B. Øksendal, T. Lindstrøm and A. S. Ustunel (eds.), Gordon and Breach Science Publishers. 1993.

[2] F. E. Benth: The Stochastic Cauchy Problem With Applications To Nonlinear Filtering. cand.scient thesis. University of Oslo 1993.

[3] G. B. Folland: Real Analysis. Wiley Interscience, 1984.

[4] H. Gjessing, H. Holden, T. Lindstrøm, B. Øksendal, J. Ubøe and T.-S. Zhang: The Wick Product. To appear in A.Melnikov(editor):New Trends in Probability and Statistics, Vol iv. TVP Publishers, Moscow.

[5] I. Gyöngy \& N. V. Krylov: SPDEs With Unbounded Coefficients I \& II; Stochastics 32-33, 1990 .

[6] T. Hida, H.-H. Kuo, J. Potthoff and L. Streit: White Noise Analysis -An Infinite Dimensional Calculus. Kluwer Academic Publishers. 1993.

[7] H. Holden, T. Lindstrøm, B. Øksendal, J. Ubøe, T.-S. Zhang: Stochastic Boundary Value Problems. A White Noise Functional Approach. Manuscript.

[8] E. Hille and R. Phillips: Functional Analysis and Semigroups. Amer. Math. Soc. Colloq. Publ. Vol xxxi, 1957.

[9] I. Karatzas \& S. Shreve: Brownian Motion and Stochastic Calculus. Springer 1988.

[10] D. Nualart \& M. Zakai: Generalized Stochastic Integrals and the Malliavin Calculus. Prob.Theory and Rel.Fields 73, pp.255-280, 1986.

[11] T. Lindstrøm, B. Øksendal and J. Ubøe: Wick-Multiplication and Ito-Skorohod Stochastic Differential Equations. In S.Albeverio et al (editors) Ideas and Methods in Mathematical Analysis, Stochastics, and Applications. Cambridge Univ. Press 1992, pp.183-206.

[12] E. Pardoux: Stochastic Partial Differential Equations and Filtering of Diffusion Processes. Stochastics 3. 1979.

[13] T.-S. Zhang: Characterizations of White Noise Test Functions and Hida Distributions. Stochastics 41, 1992.

[14] B. Øksendal : Stochastic Differential Equations. 2nd. edition. Springer 1989.

[15] B. Øksendal \& T.-S. Zhang: The Stochastic Volterra Equation. Manuscript 1992. 